\title{
Treatment and Prevention Strategies for Patients with Gynecological Malignancies During the COVID-19 Pandemic
}

This article was published in the following Dove Press journal: Risk Management and Healthcare Policy

\section{Jiang Yang* \\ Jin Liu* \\ Suting Li \\ Li Hong}

Department of Obstetrics and Gynaecology, Renmin Hospital of Wuhan University, Wuhan, People's Republic of China

*These authors contributed equally to this work
Correspondence: Li Hong

Email drhongli77@gmail.com

\begin{abstract}
COVID-19 has the characteristics of rapid onset, strong infectivity, and fast course change, which has caused a worldwide pandemic. Now, our hospital and department have implemented a series of efficacious stratagems to control the spread of the COVID-19. However, the daily-increasing cases are continuously at an extraordinary level, which mean the requirement and mission to prevent and control the pandemic are enormous and urgent. However, because of the urgency of the disease and the timeliness of treatment, gynecological tumor patients cannot be administered in the hospital until the pandemic situation could be controlled entirely. Consequently, this review proposes the following suggestions and our experience about preventing and controlling of COVID-19 and the treatment for patients with gynecological tumors in gynecology departments.
\end{abstract}

Keywords: COVID-19, prevention and control strategies, treatment, strategy

\section{Introduction}

Since the outbreak of novel coronavirus pneumonia (COVID-19) in December $2019,{ }^{1}$ as of July 21,2020 , a total of 15,063,264 cases of COVID-19 were diagnosed globally, with 234,684 new cases and more than 600,000 death cases $^{2}$. COVID-19 is characterized by rapid attack, intense infectivity, and expedited alteration of disease progression. The transmission routes that have been confirmed are droplet transmission, contact transmission, and air transmission fecal-oral transmission requires further validation. ${ }^{3}$ As a crowd gathering place, the hospital is an extremely high-risk area for virus transmission. Most gynecological cancer patients are old-aged and vulnerable, accompanied by extended primary diseases as well as weaker immune-response ability. Consequently, they are classified to the high-risk COVID-19 infection group. By contrast, considering the progression and the urgent treatment need of the disease, the therapy plan requires timely surgery or chemoradiation treatment, and cannot wait to be achieved when the COVID-19 pandemic situation was completely controlled. Since January 2020, there have been a total of 3 confirmed cases of COVID-19 infection in the department of Gynecology, Renmin hospital of Wuhan University. They were all defined as high-risk susceptible groups, and all had gynecologic malignancies and a recent history of chemotherapy. Two of the cases were the first neoadjuvant chemotherapy for the diagnosis of gynecological malignancies. Although the chemotherapy caused immunocompromise, they were screened and diagnosed as COVID-19 promptly after abnormalities were found. It 
indicated a favourable prognosis after effective and standardized treatment. By contrast, the third patient had a history of multiple chemotherapy, coupled with a very poor physical foundation, rapidly progress to respiratory and circulatory failure after the diagnosis of COVID-19 and eventually died. Therefore, this article proposes the following suggestions on how to effectively prevent and control COVID-19 in relevant departments in comprehensive hospital and perform timely and effective anti-tumor treatment for these types of patients in this particular situation.

\section{Characteristics of Patients with Gynecological Malignancies}

Gynecological malignancies include cervical cancer, ovarian cancer, endometrial cancer, and vulvar cancer, which account for an extremely high proportion of patients treated in Gynecological Departments. All malignant tumors are chronic wasting diseases, and the incidence of malnutrition is as high as $40 \%-80 \%$. Meanwhile, with the development of the disease, symptoms such as vaginal bleeding, abdominal distension, abdominal pain, and depression are gradually aggravated, which severely affects patients' lives and Quality of life. Consequently, timely and standardized surgical treatment is the most crucial part of gynecological malignancy treatment. At the same time, chemoradiotherapy or bio-targeted therapy is a necessary method for the treatment of such patients as well, but radiotherapy and chemotherapy should be continuous and time-effective. If the treatment was not completed within sufficient time, the patient's survival time would be severely affected. Besides, patients with gynecological malignancies generally have obvious psychological problems such as depression, anxiety disorder, panic disorder, and sleep disorder. To be specific, anxiety are mostly from the stress that not being administered in time before surgery, depression may be caused by genital resection. In addition, weight loss, hair loss, and somatization would be caused by side effects of drugs after surgery or after chemotherapy. ${ }^{4}$ These factors grievously influence the survival and Quality of life of patients. Accordingly, early and effective treatment is a constant stratagem to ensure the health of patients.

\section{Strengthen Patient Education When They are Admitted}

In the prevention and control of COVID-19, controlling the origin of infection and cutting off the transmission route are essential measures of controlling the epidemic situation. Admission and education for patients are necessary measures to prevent the virus from spreading among the population. According to the requirements of the "Technical Guidelines for the Prevention and Control of COVID-19 in Medical Institutions"5 combined with the particular circumstances of our department, a "COVID-19 Warm Tips Notification Letter" is prepared for recently admitted gynecological tumor patients, and patients and their families understand admission can only be processed after signing the agreement. The specific mission content is as follows: (1) After admission, patients must wear a mask consciously, and are prohibited from asking for leave. (2) After admission, patients should actively cooperate with medical staff to arrange and check, and routinely monitor the body temperature. If the body temperature exceeds $37.3{ }^{\circ} \mathrm{C}$ or patients have respiratory symptoms, inform the responsible nurse and doctor in time. (3) One person with one escort system is implemented. Patients and escorts are not permitted to go out without authorization. Relatives deliver commodities and daily meals. Non-accompanied relatives cannot enter the ward. (4) During hospitalization, it is forbidden to transfer between wards. Maintaining personal hygiene, ensuring adequate sleep, increasing nutrition, light diet, intake plenty of water, and improving autoimmunity ability should be attached importance.

\section{Admission Management of Patients with Gynecological Malignancies Admission Criteria Must Be Clarified}

According to the COVID-19 pandemic prevention and control obligations, it is necessary to avoid crowds as much as possible to reduce personnel contact activity. Comprehensive Hospitals are COVID-19 treatment centers and are composed of crowded units, eventuating that frequent entry and exit will increase the risk of COVID-19 infection. Therefore, all patients who are to be admitted to the hospital need to be diagnosed with gynecological malignant tumor (cancer) patients and need to be admitted to the hospital for surgery or radiotherapy and chemotherapy. Eliminate patients undergoing elective surgery or no hospitalization to reduce the burden of epidemic prevention and control. The most extended incubation period of COVID-19 can be up to 14 days. ${ }^{6,7}$ Consequently, all patients admitted to the hospital must adopt an online appointment system. Patients must be quarantined at 
home for 14 days before admission, and report body temperature on the appointment website every day before they can come to the hospital for admission. When admission, a Lung CT scan is required. If there are ground-glass changes, patients must be guided to a fever clinic.

\section{Develop a Zoning Diagnosis and Treatment System}

The ward implements a zoning diagnosis and treatment system, which is distributed into public areas, patient areas, and medical care areas. The patient areas are arranged in isolation wards, observation wards, transition wards, and safe wards. Besides, a single escort family and a separate room system to avoid cross-infection. The latest research reports that the median incubation period of COVID-19 is 4.75 days (interquartile range: $3.0-7.2$ ), ${ }^{8}$ so patients need to be quarantined in the observation ward for 3-5 days after admission, if without fever and COVID-19related respiratory tract they can be transferred to the transition ward. The patients in the transition ward were observed for 2-3 days and then transferred to the safe ward. Meanwhile, according to health status, a surgical plan or radio-chemotherapy plan is formulated, and all subsequent treatments will be performed in the safety ward.

\section{Formulate an Emergency Procedure}

During the hospitalization, if the patient has respiratory or gastrointestinal symptoms, the emergency procedure should be performed immediately. For patients diagnosed with COVID-19, they must be transferred to an isolation ward or COVID-19 specialist therapy according to their condition. Moreover, considering the rapid development of COVID-19, surgery or chemotherapy needs to be terminated, otherwise it will aggravate disease progression and be perniciously to patients. After the COVID-19 is cured and a multi-disciplinary treatment (MDT) is conducted, the patient will be re-evaluated whether to receive antitumor therapy or re-admitted to the hospital after recuperation. Meanwhile, all medical staff and family members who were in contact with the patients, as well as inpatients and their families who were in the same period, must be isolated, clinically observed or perform self-quarantine. On the other side, it is significant to identify and remove the origin of infection, cut off the transmission route to control nosocomial infections in the department.

\section{Clustering Intervention is Established}

Clustering intervention is a collection of evidence-based treatment and nursing measures. It follows the "EvidenceBased Practice Guidelines" and can maximize the quality of care and improve the prognosis of patients ${ }^{9}$. Initially proposed by the Institute for Healthcare Improvement (IHI), the purpose is to help medical staff to provide patients with the best achievable medical care assistance. It is now gradually extended to clinical treatment, that is, cluster treatment. From the perspective of a holistic view, it integrates independent intervention methods into a holistic "bundled treatment strategy," in order to maximize the overall treatment effect and improve the prognosis of the disease. Each clustering treatment plan includes multiple elements, each of which is clinically proven to improve the patient's prognosis, and each element is independent and highly operable. ${ }^{10}$ Now it has maturely applied to the treatment of patients with sepsis in ICU and has obtained good clinical results, which has become the focus of international guidelines for the treatment of sepsis. ${ }^{11,12}$

Patients with gynecological malignancies are mostly middle-aged and elderly people, with a high percentage of cachexia and low immunity ability. Besides, they may undergo a wide range of surgery, extended time, and significant injuries. The generally used chemotherapy drugs platinum and paclitaxel have serious side effects such as impaired gastrointestinal function and bone marrow suppression. Advanced age, tumors, surgery, and chemotherapy are all high-risk factors for venous thrombosis. Therefore, based on evidencebased medicine, the Disciplinary Committee discuss and formulate clustered nursing and treatment strategies suitable for the treatment of gynecological malignancies, in order to reduce postoperative patients with poor wound healing, lower extremity venous thrombosis, postoperative infections, and alleviate gastrointestinal symptoms in chemotherapy patients Complications such as tract response, bone marrow suppression. These are of considerable significance to promote patient recovery and shorten the length of hospital stay. It is also an effective measure to prevent nosocomial infection in COVID-19 and control the spread of the pandemic.

\section{Identification of Fever After Surgery or Chemotherapy and COVID-I9}

According to the NCCN guidelines, the principle of treatment of gynecological malignancies is effective radical surgery combined with chemoradiation. However, 
weakened immune systems due to postoperative infection and bone marrow suppression after chemotherapy has always been one of the difficulties affecting the quality of life of patients. Studies illustrated that the postoperative infection rate of gynecological malignancies is $19.67 \%$, of which urinary tract infection, respiratory tract infection, genital tract infection, surgical incision infection, and gastrointestinal tract infection respectively account for $50.00 \%, 25.00 \%, 12.50 \%, 8.33 \%$, and $4.17 \%[13]$, while bone marrow suppression caused by chemotherapy can reach up to $59.2 \%[14]$. Regardless of postoperative infection or myelosuppression after chemotherapy, the most common accompanying symptoms are fever, loss of appetite, and malaise. Most of the first symptoms of COVID-19 are fever, and a small part of them will also reveal gastrointestinal symptoms. Importantly, how to correctly distinguish the complications after surgery or chemotherapy and COVID-19 infection is of great significance for controlling the development of the pandemic and preventing the virus from spreading in the hospital.

\section{Outpatient Management}

Patients are fragile and with weakened immune systems, and have not fully recovered from the impaired gastrointestinal function after surgery or after chemotherapy. During the pandemic, verbal and written discharge propaganda and education declaration are necessary. The details are as follows. Utilize a healthy diet, ensure drinking more water, and intake more nutrients to promote physical recovery. Pay attention to mental health, such as keeping positive and optimistic. Do proper exercise to promote or restore gastrointestinal function and prevent venous thrombosis. Within 2 weeks after discharge, limit the intensity and scale of activity, reduce contact with non-residents of the family, and try to keep one person assigned to caring. Ventilate 2-3 times a day in the home, and the ventilation time is not less than 30 minutes each time. A Face mask is necessary if one family person goes out in weeks after leaving the hospital. Wash hands, change clothes, and dry clothes in a ventilated place after going home. Use $75 \%$ alcohol or chlorine-containing disinfectant for disinfection indoors. Visit the hospital in time if there are respiratory symptoms such as fever, cough, and malaise, difficulty eating, abdominal distension, abdominal pain.

\section{Summary}

The above-mentioned highlights illustrated the prevention strategies for COVID-19 in patients with gynecological tumors during admission, hospitalization and discharge. However, how to balance the urgency of treatment for patients with tumors (cancers) and the requirement for COVID-19 protection is an extraordinarily vital issue. Therefore, the management strategy of gynecologic malignancies patients during the epidemic should consider the following three factors. (1) Epidemiological situation of patients and hospitals: in areas with severe pandemics, we should first strictly prevent infection and epidemic spread, and then wait for the opportunity to carry out anti-tumorrelated treatment. In lower areas, it is recommended to ensure timely treatment of patients with malignant tumors, while doing well in pandemic prevention and control. (2) The specific types of tumors and the medical resources currently available: for ovarian cancer, endometrial cancer, and early cervical cancer, which are mainly treated by surgery, elective surgery should be arranged as soon as possible when the pandemic situation permits. Considering that surgical resources are relatively scarce in some areas, or pandemic prevention and control requirements are temporarily inoperable, alternative treatment options such as neoadjuvant chemotherapy can be selected without violating the principles of tumor treatment. Therefore, when the first-line treatment of tumors cannot be satisfied due to epidemic prevention and control, the treatment strategy should be adjusted in time, and other alternative treatment options should be used. (3) Establish effective communication methods: doctors can maintain communication with patients through "telemedicine and remote counselling" and predict patients' condition. When evaluated nonemergency surgeons, patients are advised to postpone elective surgery. Patients who are assessed as scheduled treatment should postpone chemotherapy or surgery properly after weighing the risk of infection and tumor. Patients who must undergo chemotherapy or surgery after prejudged can be admitted to the hospital as soon as possible under the condition of proper protections for doctors and patients. If the patient's condition worsens, seeking medical treatment under the guidance of an evaluation doctor was recommended for them.

The comprehensive hospital is the principal battlefield of anti-pandemic diseases. In addition to the departments of infection, respiratory, and critical medicine, other departments have been deployed to support the rescue work of the COVID-19 isolation ward. In addition, all hospital materials are provided priority to supply the frontline department. Therefore, the shortage of gynecological 
medical staff and the relative lack of medical resources and protective equipment have brought significant challenges to the treatment of patients with gynecological malignancies. Gynecology needs to optimize the treatment of patients with the tumor and effectively prevent and control nosocomial infections with COVID-19.

\section{Acknowledgments}

We would like to thank everyone from the Department of Obstetrics and Gynecology, Renmin Hospital of Wuhan University, for their sincere help and technique support. This research is sponsored by Hubei Province's Outstanding Medical Academic Leader Program.

\section{Disclosure}

The authors report no conflicts of interest in this work.

\section{References}

1. Zhu N, Zhang D, Wang W, et al. A novel coronavirus from patients with pneumonia in China, 2019. N Engl J Med. 2020;382(8):727-733. doi:10.1056/NEJMoa2001017

2. WHO. Coronavirus disease 2019 (COVID-19) situation report; July 21, 2020. Available from: http://www.securitymanagers.net/who-covid -19-situation-report/. Accessed August 5, 2020.

3. Commission $\mathrm{CNH}$. The guidelines for diagnosis and treatment of COVID-19 (the seventh edition draft); 2020. Available from: http:// www.nhc.gov.cn/xcs/zhengcwj/202003/46c9294a7dfe4cef80dc7 f5912eb1989.shtml. Accessed August 5, 2020.
4. Chan YM, Lee PWH, Fong DYT, et al. Effect of individual psychological intervention in Chinese women with gynecologic malignancy: a randomized controlled trial. J Clin Oncol. 2005;23(22):4913-4924. doi:10.1200/JCO.2005.02.069

5. Commission $\mathrm{CNH}$. The guidelines for the prevention and control of COVID-19 in medical institutions (the first edition draft); March 04, 2020. Available from: http://www.nhc.gov.cn/xcs/zhengcwj/202001/ b91fdab7c304431eb082d67847d27e14.shtml. Accessed August 5, 2020.

6. Huang C, Wang Y, Li X, et al. Clinical features of patients infected with 2019 novel coronavirus in Wuhan, China. Lancet. 2020;395 (10223):497-506. doi:10.1016/S0140-6736(20)30183-5

7. Zhou F, Yu T, Du R, et al. Clinical course and risk factors for mortality of adult inpatients with COVID-19 in Wuhan, China: a retrospective cohort study. Lancet Neurol. 2020;395(10229):1054-1062. doi:10.1016/ S0140-6736(20)30566-3

8. Chen N, Zhou M, Dong X, et al. Epidemiological and clinical characteristics of 99 cases of 2019 novel coronavirus pneumonia in Wuhan, China: a descriptive study. Lancet Neurol. 2020;395(10223):507-513. doi:10.1016/S0140-6736(20)30211-7

9. Crocker C, Kinnear W. Weaning from ventilation: does a care bundle approach work? Intensive Crit Care Nurs. 2008;24(3):180-186. doi:10.1016/j.iccn.2007.11.003

10. Marra A, Ely EW, Pandharipande PP, Patel MB. The ABCDEF bundle in critical care. Crit Care Clin. 2017;33(2):225-243. doi:10. 1016/j.ccc.2016.12.005

11. Mukherjee V, Evans L. Implementation of the surviving sepsis campaign guidelines. Curr Opin Crit Care. 2017;23(5):412-416. doi:10. 1097/MCC.0000000000000438

12. Levy MM, Pronovost PJ, Dellinger RP, et al. Sepsis change bundles: converting guidelines into meaningful change in behavior and clinical outcome. Crit Care Med. 2004;32(11 Suppl):S595-S597. doi:10. 1097/01.CCM.0000147016.53607.C4
Risk Management and Healthcare Policy

\section{Publish your work in this journal}

Risk Management and Healthcare Policy is an international, peerreviewed, open access journal focusing on all aspects of public health, policy, and preventative measures to promote good health and improve morbidity and mortality in the population. The journal welcomes submitted papers covering original research, basic science, clinical \& epidemiological studies, reviews and evaluations,

\section{Dovepress}

guidelines, expert opinion and commentary, case reports and extended reports. The manuscript management system is completely online and includes a very quick and fair peer-review system, which is all easy to use. Visit http://www.dovepress.com/testimonials.php to read real quotes from published authors. 